\title{
Image Quality Assessment for Blurred Images Using Nonsubsampled Contourlet Transform Features
}

\author{
Kanjar De*, Masilamani V. \\ Indian Institute of Information Technology, Design and Manufacturing-Kancheepuram, Chennai 600127, \\ India. \\ * Corresponding author. Tel: +91-44-27476346; email: coe10d001@iiitdm.ac.in \\ Manuscript submitted November 2, 2015; accepted December 27, 2015. \\ doi: $10.17706 /$ jcp.12.2.156-164
}

\begin{abstract}
One of the most common distortions in images is blur which makes performing lot of image processing and computer vision tasks like detection and recognition a very difficult task. Assessment of quality images corrupted blur is very important and active area of research. Nonsubsampled contourlet transform is one of the transformation which is shift invariant and used in lot of tasks of image processing like denoising, image enhancement etc. In this paper we propose an image quality measure for blurred images which uses non subsampled contourlet transform (NSCT) features and support vector regression for the task of image quality assessment of images corrupted by blur. Comparative studies with other well known image sharpness and quality measures on standard databases and correlations with Human visual system (HVS) is also presented in the paper.
\end{abstract}

Key words: Blur, human visual system(HVS), image sharpness, image quality assessment(IQA), no-reference image quality assessment (NR-IQA).

\section{Introduction}

Image quality assessment is currently one of the important areas of research in field of image processing. Images can be distorted due to various sources during image acquisition, image compression or transmission through a communication channel. Additive noise, blur, compression artifacts are examples of some of the most common distortions. Image quality assessment algorithms are divided into three categories:

1) Full Reference Image Quality Assessment (FR-IQA) 2) Reduced Reference Image Quality Assessment (RR-IQA) 3) No Reference Image Quality Assessment (NR-IQA) [1]. Full Reference image quality assessment requires a reference image of the same scene to assess quality of a distorted image. Peak Signal to Noise Ratio [2] (PSNR), Structural Similarity Index Measure [3] (SSIM) and Feature Similarity Index Measure (FSIM) [4] are classical examples of full reference image quality assessment algorithms. Reduced reference image quality assessment techniques also require a reference image of the same scene but instead of full image information of the reference image for task of image quality assessment, only a few features of the reference image is used for the task of quality assessment. Reduced Reference Entropic Differencing [5] and Reduced-reference image quality assessment by structural similarity estimation [6] are couple of the recent well known technique in this category. The final class of image quality assessment algorithms give an estimate of image quality lindly without the use of reference images and termed as no reference image quality assessment algorithms. 
Image quality assessment techniques are either designed for general cases which works for a few distortion types or for a specific type of distortion. Specific techniques for noise [7]-[9], JPEG compression artifacts [10], [11], JPEG2000 compression artifacts [12]. One of the strategy of designing image quality assessment algorithms is to extract statistics of image in spatial domain or some transform domain like wavelet, curvelet etc. Another common strategy for designing image quality assessment algorithms is to use some machine learning algorithms support vector regression, general regression neural network [13], random forest [14] regression etc. Image sharpness assessment is an active area pursued by researchers working in the area of image quality assessment. Blurring in images can be caused by a variety of sources like camera shake, defocus. In presence of excessive blur in images, tasks of object detection and recognition becomes extremely difficult. Analysis of image sharpness is an extremely important operation for image processing algorithms and lot of research is happening in this area. In this paper, we extract non subsampled contourlet transform features and develop a support vector regression based model for task of sharpness/blurriness assessment. tate of the art algorithms are proposed in literature for image quality assessment images for blurred images. Some of the common examples are based on the concept of Just Noticeable Blur(JNB) [15], Cumulative Probability of Blur Detection(CPBD) [16], edge width (referred as PINA in rest of the paper) [17], frequency domain [18], wavelet based algorithm FISH and block based

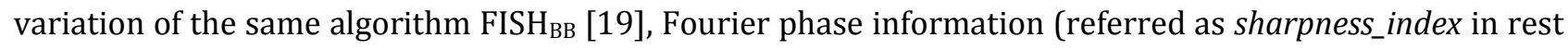
of the paper) [20], global phase coherence (referred as $s_{-}$index in rest of the paper) [21]. The rest of the paper is organized as follows Section 2 gives a brief description of the non subsampled contourlet transform, followed by Section 3 where we propose the implementation details of the measure, followed by results and discussion in Section 4 and finally we conclude in Section 5.

\section{Nonsubsampled Contourlet Transform}

Researchers working in the field of image processing use non subsampled contourlet transform for a variety of applications like image denoising, image enhancement etc. One of the most important properties of non subsampled contourlet transform is that this transform is fully shift invariant, the next property is that it is multiscale. The structure of non subsampled contourlet transform is provided in Fig. 1. Image quality assessment using non subsampled contourlet transform has been proposed earlier [22]. The details of different variants of implementation and its applications are described in [23]. Let $I(x, y)$ be an image where $0<x \leq M$ and $0<y \leq N$. $L$ denote the number of scales, $I$ is transformed resulting in $L$ bandpass subbands and one lowpass subband. Further each of the bandpass subband is decomposed into nonsubsampled directional filter banks (NSDFB). The number of NFSDB are $2^{k_{l}}$ where $1<l \leq L$. The equation of decomposition is given by

$$
C=\left\{C_{j} \mid j=1,2, \ldots,\left(\sum_{l=1}^{L} 2^{k_{l}}+1\right)\right\}
$$

where $C_{1}$ is the lowpass subband, and $C_{j}$, where $j=2, \ldots .,\left(\sum_{l=1}^{L} 2^{k_{l}}+1\right)$ are the bandpass directional subbands filtered by nonsubsampled directional filter banks. For each subband $C_{j}$, the frequency coefficient of a given pixel $(x, y)$ is represented by $C_{j}(x, y), j=1,2, \ldots,\left(\sum_{l=1}^{L} 2^{k_{l}}+1\right)$.

Multiscale and directional decomposition stages in non subsampled contourlet transform (NSCT) are independent of each other. For this reason, we have considered each band as independent and extracted individual features from each band (Fig. 1). 


\section{Proposed Technique}

The overall model is shown in Fig. 2.

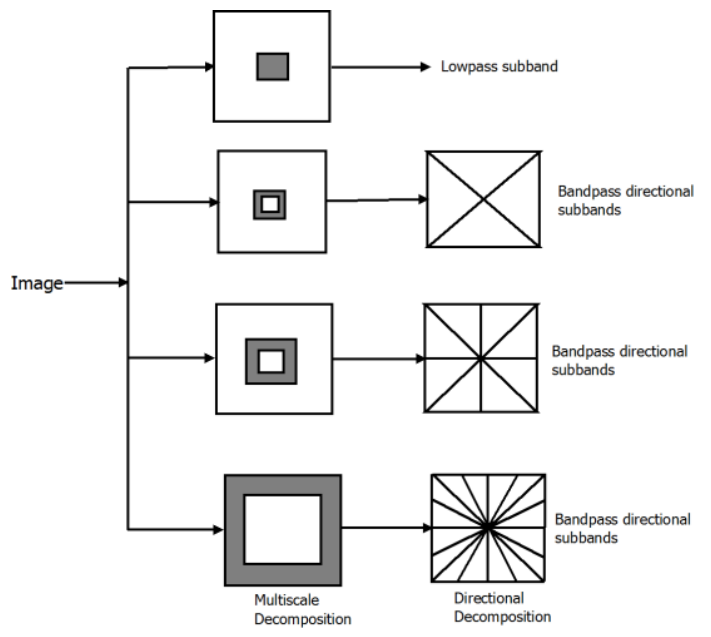

(a) Block diagram of NSCT decomposition.

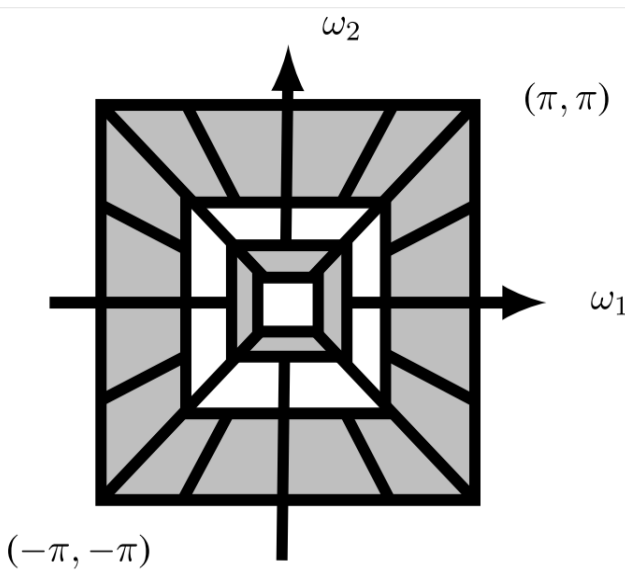

(b) Frequency division.

Fig. 1. The non subsampled contourlet transform [22].

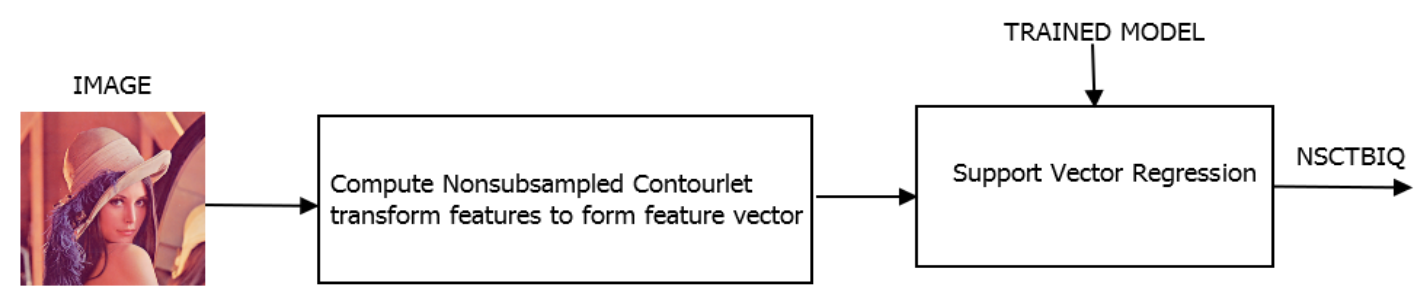

Fig. 2. Block Diagram of the proposed model.

\subsection{Extraction of Features}

As we have discussed earlier non subsampled contourlet decomposition of a 2D image into 4 scales produces 16 directional subbands. We take mean coefficient value of one subband as feature, therefore 16 subbands will yield 16 features. Now we have obtained a feature vector of dimension 16 . The filters chosen for decomposition for the proposed method are "dmaxflat7 " and "maxflat" filters. These features contain information about image sharpness.

\subsection{Support Vector Regression}

From feature space to image quality score is achieved by a support vector regression. Support Vector regression(SVR) approach is widely used for image quality assessment. Few of the well known techniques using SVR are [24]-[26]. For our implementation, we have used the LIBSVM [27] package for Support vector regression. The fourth level decomposition of an image gives 16 directional sub bands. The mean coefficients of each subband is used as a feature in this proposed technique. Support vector regression model is trained using this technique. Support vector regression model is trained using these features and DMOS score available with the dataset. We use $\varepsilon$-SVR for our model. Radial Basis function (RBF) kernel is used as the kernel function.

\subsection{Selection of Features}

For machine learning problems, having a long feature vector leads to complications which affects both performance and accuracy. To combat this challenge we need to use some feature selection algorithm which reduces the dimensionality of the feature vector and try to improve the performance of the proposed 
model. Sequential forward feature selection algorithm is used in the proposed technique to select the best features. This type of algorithm is used in image quality assessment algorithms [28] for selecting features for multimethod fusion.

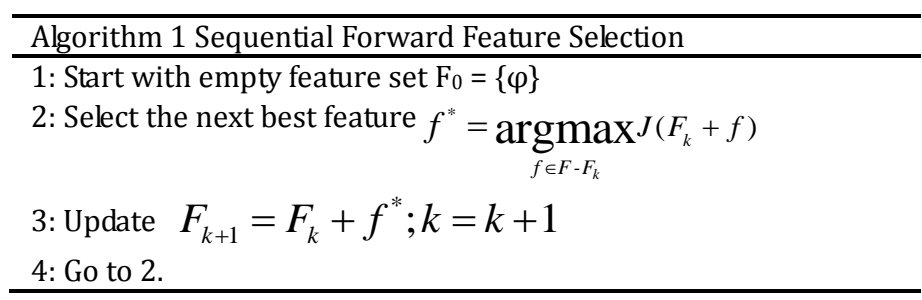

Let $F=\left\{f_{j} \mid j=1, \ldots, 16\right\}$ be the feature set with 16 features, goal is to find a subset $F_{N}$, with $N<16$ to optimize an objective function $J\left(F_{k}\right)$ which can be one of the following three functions.

$$
\begin{gathered}
J\left(F_{N}\right)=\operatorname{SRCC}\left(\operatorname{NSCTBIQ}\left(F_{N}\right), \operatorname{DMOS}_{d}\right) \\
J\left(F_{N}\right)=\operatorname{PLCC}\left(\operatorname{NSCTBIQ}\left(F_{N}\right), \operatorname{DMOS}_{d}\right) \\
J\left(F_{N}\right)=\operatorname{RMSE}\left(\operatorname{NSCTBIQ}\left(F_{N}\right), \operatorname{DMOS}_{d}\right)
\end{gathered}
$$

where NSCTBIQ stands for non subsampled contourlet transform blurred image quality score, $D M O S_{d}$ is Differential Mean Opinion Score of a database d, SRCC is Spearman Rank Order Correlation Coefficient, PLCC is Pearson Linear Correlation Coefficient and RMSE is Root mean square Error. For equations (2) and (3), maximization is done and for equation (4) minimization of cost must be done. Spearman Rank Order Correlation Coefficient is defined as

$$
S R C C=1-\frac{6}{N\left(N^{2}-1\right)} \sum_{i=1}^{N} d_{i}^{2}
$$

where $d_{i}$ is the difference between the subjective DMOS rank and objective image quality score rank of the $i^{\text {th }}$ image of the database, $i=1,2, \ldots . . N$. Pearson Linear Correlation Coefficient is defined as

$$
P L C C=\frac{\sum_{i}\left(x_{i}-\bar{x}\right)\left(y_{i}-\bar{y}\right)}{\sqrt{\left(x_{i}-\bar{x}\right)^{2}\left(y_{i}-\bar{y}\right)^{2}}}
$$

where $x_{i}$ is the subjective DMOS score of the $i^{\text {th }}$ image and $\mathrm{y}_{\mathrm{i}}$ is the objective image quality score of the $i^{\text {th }}$ image, $\bar{x}$ is the mean subjective DMOS score of the dataset $D$ and $\bar{y}$ are the mean objective image quality score of dataset $D, i=1,2, \ldots, N, N=$ total number of images in the dataset $D$. Root mean Square Error is defined as

$$
R M S E=\sqrt{\frac{1}{N} \sum\left(x_{i}-y_{i}\right)^{2}}
$$

where $N$ is the total number of images in dataset $D, x_{i}$ is the subjective DMOS score of the $i^{\text {th }}$ image and $\mathrm{y}_{\mathrm{i}}$ is the objective image quality score of the $i^{\text {th }}$ image.

\section{Results and Discussion}

To validate our proposed method we validate it with publicly available standard datasets for image quality assessment. We perform two types of statistical analysis for validation of our proposed system against Human visual system (HVS). The first type of analysis is to evaluate prediction 
monotonicity-Spearman Rank Order Correlation Coefficient (SROCC) mentioned in equation(5) is a metric used for evaluating Prediction monotonicity. Higher the value of SROCC, it is more closer to human visual perception. The next type of analysis is to evaluate prediction accuracy- Pearson Linear Correlation Coefficient (PLCC) mentioned in equation(6) and Root Mean Square Error (RMSE) mentioned in equation(7) are the metrics used for evaluation of prediction accuracy. High value of PLCC denotes that the proposed model is close to the human visual system. For RMSE, a lower value means it closer to the human visual system. As per recommendations from Video Quality Experts Group (VQEG) report the image quality score must be passed through a non linear regression function. [29].

$$
Q_{p}=\beta_{1}\left(\frac{1}{2}-\frac{1}{\exp \left(\beta_{4}\left(Q-\beta_{3}\right)\right)}\right)+\beta_{4} Q+\beta_{5}
$$

where $\beta_{1}, \beta_{2}, \beta_{3}, \beta_{4}, \beta_{5}$ are regression parameters, $Q$ and $Q_{P}$ are the predicted image quality scores before and after regression respectively. For our analysis, we have split the entire data into training and testing set in the ratio $80 / 20$ where 80 per cent of data is used for training and 20 per cent is used for testing. 1000 different combinations of train-test sets are generated for running experiments. We use medianvalues of the metrics for our performance analysis. We have compared our proposed technique against PSNR, SSIM, JNB, CPBD, FISH, FISH ${ }_{\mathrm{BB}}$, Q-metric, PINA and the techniques proposed using phase coherence (Sharpness_Index and S_index) for our analysis.

\subsection{LIVE Database [30]}

From 29 reference images 145 blurred images are created in this database. Table 1 shows the performance of proposed technique against state of the full reference and no reference image quality assessment and image sharpness assessment techniques. We see that the proposed approach is comparable to the No-reference Image Quality assessment algorithms and performs better than Full reference techniques like PSNR, SSIM for this dataset.

Table 1. Performance-LIVE Database

\begin{tabular}{|c|c|c|c|c|}
\hline Technique & Type & SRCC & PLCC & RMSE \\
\hline Proposed NSCTBIQ & No-Reference & 0.9102 & 0.9312 & 5.5674 \\
\hline PSNR & Full Reference & 0.7765 & 0.8110 & 8.8767 \\
\hline SSIM & Full Reference & 0.892 & 0.8954 & 6.8022 \\
\hline JNB & No-Reference & 0.7823 & 0.8437 & 8.1897 \\
\hline CPBD & No-Reference & 0.9131 & 0.9308 & 5.5402 \\
\hline FISH & No-Reference & 0.8731 & 0.9133 & 6.1505 \\
\hline $\mathrm{FISH}_{\mathrm{BB}}$ & No-Reference & 0.9308 & 0.9512 & 4.4682 \\
\hline$Q$-metric & No-Reference & 0.5596 & 0.7349 & 10.3576 \\
\hline PINA & No-Reference & 0.8962 & 0.9307 & 5.5725 \\
\hline Sharpness Index & No-Reference & 0.8538 & 0.8102 & 8.5319 \\
\hline S Index & No-Reference & 0.8547 & 0.8104 & 8.5826 \\
\hline
\end{tabular}

\subsection{VCLFER Database [31]}

This database consists of 138 blurred images created from 23 reference images Table 2 shows the performance of proposed technique against state of the full reference and no reference image quality assessment and image sharpness assessment techniques. We have compared our technique with available techniques in the literature and have noticed that the proposed technique performs better than the well known algorithms like JNB and CPBD, but the metric proposed in [17] gives a slightly better performance in this dataset. 


\subsection{CSIQ Database[32]}

From 30 reference images 150 blurred images are created in this database. Table 3 shows the performance of proposed technique against state of the full reference and no reference image quality assessment and image sharpness assessment techniques. For CSIQ dataset this performs better than most of the standard no-reference image sharpness technique except the block wise version of FISH which gives a slightly better performance for this particular dataset.

Table 2. Performance-VCLFER Database

\begin{tabular}{|l|l|l|l|l|}
\hline Technique & Type & \multicolumn{1}{l|}{ SRCC } & PLCC & RMSE \\
\hline Proposed NSCTBIQ & No-Reference & 0.8656 & 0.9113 & 9.7573 \\
\hline PSNR & Full Reference & 0.7757 & 0.8227 & 13.3182 \\
\hline SSIM & Full Reference & 0.8019 & 0.8609 & 11.9965 \\
\hline JNB & No-Reference & 0.7411 & 0.7802 & 14.8299 \\
\hline CPBD & No-Reference & 0.8609 & 0.9142 & 9.5635 \\
\hline FISH & No-Reference & 0.8236 & 0.8866 & 10.9253 \\
\hline FISHBB & No-Reference & 0.7945 & 0.8606 & 11.9063 \\
\hline Q-metric & No-Reference & 0.5212 & 0.6051 & 10.3576 \\
\hline PINA & No-Reference & 0.8775 & 0.9197 & 9.2068 \\
\hline Sharpness Index & No-Reference & 0.7816 & 0.6455 & 17.9634 \\
\hline S index & No-Reference & 0.7945 & 0.6457 & 18.020 \\
\hline
\end{tabular}

Table 3. Performance-CSIQ Database

\begin{tabular}{|l|l|l|l|l|}
\hline Technique & Type & SRCC & PLCC & RMSE \\
\hline Proposed NSCTBIQ & No-Reference & 0.8651 & 0.8898 & 0.1278 \\
\hline PSNR & Full Reference & 0.9155 & 0.9112 & 0.1168 \\
\hline SSIM & Full Reference & 0.9248 & 0.9190 & 0.1105 \\
\hline JNB & No-Reference & 0.8171 & 0.8555 & 0.1440 \\
\hline CPBD & No-Reference & 0.8548 & 0.8870 & 0.1238 \\
\hline FISH & No-Reference & 0.8596 & 0.8876 & 0.1287 \\
\hline FISH $B$ B & No-Reference & 0.8819 & 0.9051 & 0.1197 \\
\hline Q-metric & No-reference & 0.6305 & 0.6514 & 0.2128 \\
\hline PINA & No-Reference & 0.8436 & 0.8315 & 0.1566 \\
\hline Sharpness Index & No-Reference & 0.8254 & 0.6593 & 0.2114 \\
\hline S Index & No-Reference & 0.8251 & 0.6592 & 0.2117 \\
\hline
\end{tabular}

Table 4. Performance-TID2013 Database

\begin{tabular}{|l|l|l|l|l|}
\hline Technique & Type & SRCC & PLCC & RMSE \\
\hline Proposed NSCTBIQ & No-Reference & 0.8568 & 0.8792 & 0.5865 \\
\hline PSNR & Full Reference & 0.9546 & 0.9614 & 0.3399 \\
\hline SSIM & Full Reference & 0.9554 & 0.9673 & 0.3112 \\
\hline JNB & No-Reference & 0.7876 & 0.8316 & 0.6873 \\
\hline CPBD & No-Reference & 0.8446 & 0.8779 & 0.5879 \\
\hline FISH & No-Reference & 0.7855 & 0.8292 & 0.6857 \\
\hline FISHBB & No-Reference & 0.8262 & 0.8622 & 0.6240 \\
\hline Q-Metric & No-Reference & 0.3031 & 0.2985 & 1.1657 \\
\hline PINA & No-Reference & 0.7817 & 0.8247 & 0.7025 \\
\hline Sharpness Index & No-Reference & 0.8691 & 0.7482 & 0.8140 \\
\hline S Index & No-Reference & 0.8677 & 0.7478 & 0.8149 \\
\hline
\end{tabular}

\subsection{TID2013 Database [33], [34]}

From 25 reference images 125 blurred images are created in this database. Table 4 shows the performance of proposed technique against state of the full reference and no reference image quality 
assessment and image sharpness assessment techniques. Phase coherence based techniques [20], [21] perform slightly better.

\section{Conclusion}

In this paper we propose a machine learning based image quality measure for blurred images using non subsampled contourlet transform features. We use support vector regression (SVR) for combining the features and giving an estimate of image quality of blurred images. The proposed measure is comparable with the human visual system (HVS) as we have shown in the results. One of the limitations is that the feature selection algorithm used here is sequential forward selection algorithm, is useful when number of features is less, for higher number of features the algorithm is very slow. Heuristic approaches like genetic algorithms, simulated annealing etc. might offer a faster and efficient way to select desired features.

\section{References}

[1] Wang, Z., \& Bovik, A. C. (2006). Modern Image Quality Assessment, San Rafael: Morgan \& Claypool.

[2] Eskicigolu, A. M., \& Fisher, P. S. (1995). Image quality measures and their performance. IEEE Transactions on Communications, 43(12), 2959-2965.

[3] Wang, Z., Bovik, A. C., Sheikh, H. R., Simoncelli, E. P., et al. (2004). Image quality assessment: From error visibility to structural similarity. IEEE Transactions on Image Processing, 13(4), 600-612.

[4] Zhang, L., Zhang, L., Mou, X., Zhang, D., et al. (2011). Fsim: A feature similarity index for image quality assessment. IEEE Transactions on Image Processing, 20(8), 2378-2386.

[5] Soundarajan, R., \& Bovik, A. C. (2012). Rred indices: Reduced reference entropic differencing for image quality assessment. IEEE Transactions on Image Processing, 21(2), 517-526.

[6] Rehman, A., \& Wang, Z. (2012). Reduced-reference image quality assessment by structural similarity estimation. IEEE Transactions on Image Processing, 21(8), 3378-3389.

[7] Pyatykh, S., Hesser J., \& Zheng, L. (2013). Image noise level estimation by principal component analysis. IEEE Transactions on Image Processing, 22(12), 5226-5237.

[8] Liu, X., Tanaka, M., \& Okutomi, M. (2013). Single-image noise level estimation for blind denoising. IEEE Transactions on Image Processing, 22(2), 687-699.

[9] Zoran, D., \& Weiss, Y. (2009). Scale invariance and noise in natural images. IEEE Proceedings of the 12th International Conference on Computer Vision (pp. 2209-2216).

[10] Wang, Z., Sheikh, H. R. \& Bovik, A. C. (2002). No-reference perceptual quality assessment of jpeg compressed images. IEEE Proceedings of the 2002 International Conference on Image Processing (pp. 1-477).

[11] Golestaneh, S. A., \& Chandler, D. M. (2014). No-reference quality assessment of jpeg images via a quality relevance map. IEEE Signal Processing Letters, 21(2), 155-158.

[12] Sheikh, H. R., Bovik A., C. \& Cormack, L. (2005). No-reference quality assessment using natural scene statistics: Jpeg 2000. IEEE Transactions on Image Processing, 14(11), 1918-1927.

[13] Li, C., Bovik A., C. \& Wu, X. (2011). Blind image quality assessment using a general regression neural network. IEEE Transactions on Neural Networks, 22(5), 793-799.

[14] Pei, S.-C., \& Chen, L.-N. (2015). Image quality assessment using human visual dog model fused with random forest. IEEE Transactions on Image Processing, 24(11), 3282-3292.

[15] Ferzli, R., \& Karam, L. J., (2009). A no-reference objective image sharpness metric based on the notion of just noticeable blur (jnb). IEEE Transactions on Image Processing, 18(4), 717-728.

[16] Narvekar, N. D., \& Karam, L. J. (2011). A no-reference image blur metric based on the cumulative probability of blur detection (cpbd). IEEE Transactions on Image Processing, 20(9), 2678-2683. 
[17] Marziliano, P., Dufaux, F., Winkler, S., Ebrahimi, E., et al. (2002). A no-reference perceptual blur metric. Proceedings of the 2002 International Conference on Image Processing (pp. 3-57).

[18] De, K., \& Masilamani, V. (2013). Image sharpness measure for blurred images in frequency domain. Proceedia Engineering, 64, 149-158.

[19] Vu, P. V., \& Chandler, D. M. (2012). A fast wavelet-based algorithm for global and local image sharpness estimation. IEEE Signal Processing Letters, 19(7), 423-426.

[20] Leclaire, A., \& Mosian, L. (2015). A No-reference image quality assessment and blind deblurring with sharpness metrics exploiting fourier phase information. Journal of Mathematical Imaging and Vision, 52(1), 145-172.

[21] Blanchet, G., \& Mosian, L. (2012). An explicit sharpness index related to global phase coherence. IEEE Proceedings of the 2012 International Conference on Acoustics, Speech and Signal Processing (ICASSP) (pp. 1065-1068).

[22] Lu, F., Zhao, Q., \& Yang, G. (2011). Nonsubsampled contourlet transform-based algorithm for no-reference image quality assessment. Optical Engineering, 50(6).

[23] Da Cunha, A. L., Zhou, J, \& Do, M. N. (2006). The nonsubsampled contourlet transform: theory, design, and applications. IEEE Transactions on Image Processing, 15(10), 3089-3101.

[24] Moorthy, A. K., \& Bovik, A. C. (2010). A two-step framework for constructing blind image quality indices. IEEE Signal Processing Letters, 17(5), 513-516.

[25] Saad, M. A., Bovik, A. C., \& Charrier, C. (2012). Blind image quality assessment: A natural scene statistics approach in the dct domain. IEEE Transactions on Image Processing, 21(8), 3339-3352.

[26] Moorthy, A. K., \& Bovik, A. C. (2011). A Blind image quality assessment: From natural scene statistics to perceptual quality. IEEE Transactions on Image Processing, 20(12), 3350-3364.

[27] Chang, C.-C., \& Lin, C.-J. (2011). Libsvm: A library for support vector machines. ACM Transactions on Intelligent Systems and Technology, 20(12), 27-27.

[28] Liu, T.-J., Lin, W., \& Kuo, C.-C. J. (2013). Image quality assessment using multi-method fusion. IEEE Transactions on Image Processing, 22(5), 1793-1807.

[29] Final Report from the Video Quality Experts Group on the Validation of Objective Models of Video Quality Assessment. (2003). Phase ii (fr tv2).

[30] Sheikh, H. R., Wang, Z., Cormack, L., \& Bovik, A. C., (2005). The Live image quality assessment database release 2. Retrieved from http://live.ece.utexas.edu/research/quality/release2/databaserelease2.zip

[31] Zaric, A, Dumic, E., Grgic, S., et al. (2012). Vcl@ fer image quality assessment database. AUTOMATIKA: ‘casopis za automatiku, mjerenje, elektroniku, rcunarstvo i komunikacije, 53(4), 344-354.

[32] Larson, E. C., \& Chandler, D. M. (2010). Most apparent distortion: Full-reference image quality assessment and the role of strategy. Journal of Electronic Imaging, 19(1).

[33] Ponomarenko, N., Jin, L., Ieremeiev, O., et al. (2015). Image quality assessment: from error visibility to structural similarity. Signal Processing: Image Communication, 30, 57-77.

[34] Ponomarenko, N., Ieremeiev, O., Lukin, V., et al. (2013). Color image database tid2013: Peculiarities and preliminary results. IEEE Proceedings of the 2013 4th European Workshop on Visual Information Processing (pp. 106-111).

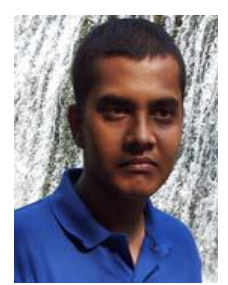

Kanjar De was born in Kolkata, India. He received the B.Tech degree in electronics and communication engineering from Sikkim Manipal Institute of Technology, Majitar, India in 2007 and the M.Tech degree from International Institute of Information Technology, Bangalore, India in 2009. He joined Indian Institute of Information Technology, Design and Manufacturing Kancheepuram, India as a Ph.D. scholar in the Department of Computer 
Science. He has one year's industrial experience. His research interests include image processing, computer vision.

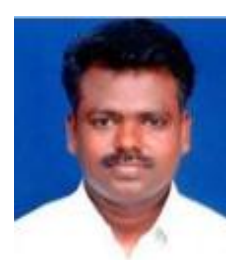

V. Masilamani is currently Assistant Professor in the Indian Institute of Information Technology, Design and Manufacturing, Kancheepuram, India. He is currently a faculty in the Department of Computer Science. He received his M.Tech degree from Indian Institute of Technology, Kharagpur, India. He completed his Ph.D. from Indian Institute of Technology, Madras, India. His research interest includes image processing and theoretical computer science. He has 12 years' teaching experience and ten years' research experience. He has a number of international journal and conference publications. 